\title{
Mandibular Movements Identify Respiratory Efforts Due to Obstructive Sleep Apnoea in a Pre-school Child
}

\author{
Jean B. Martinot ${ }^{1, *}$, Stéphane J. Denison ${ }^{1}$, Frédéric H. Senny ${ }^{2}$, Thibert A. Robillard ${ }^{1}$, Umakanth \\ Khatwa $^{3}$ and Hervé Guenard ${ }^{4}$
}

\author{
${ }^{1}$ Department of Pneumology, Ste-Elisabeth Hospital, Namur, Belgium \\ ${ }^{2}$ Electronic unit, HELMo - Gramme, Liège, Belgium \\ ${ }^{3}$ Division of Respiratory Diseases, Children's Hospital, Boston, United States \\ ${ }^{4}$ Laboratoire de Physiologie, Bordeaux University, France
}

\begin{abstract}
Adenotonsillar hypertrophy is a major cause of obstructive sleep apnea (OSAS) in childhood. Increased respiratory effort associated with OSAS is accompanied by an increase in pulse transit time (PTT) but also mandibular movements (MMs) amplify with increased upper airway resistance. We compared dynamic changes in PTT and MMs using a magnetic distance sensor during polysomnography (PSG) in a pre-school child with severe OSAS before and after adenotonsillectomy. The results show that repetitive respiratory effort to overcome upper airway obstruction can be identified in children using MMs.
\end{abstract}

Keywords: Adenotonsillar hypertrophy, mandibular movements, obstructive sleep apnea in children, pulse transit time.

\section{INTRODUCTION}

Mouth-opening and oral breathing are common clinical features during sleep in children particularly in the presence of upper airway obstruction e.g. related to adenotonsillar hypertrophy [1]. The increased upper airway resistance results in increased respiratory effort (RE) and respiratoryeffort-related arousals (RERAs). Periods of increased RE while difficult to assess are critical in identifying OSAS in children $[1,2]$.

EEG-arousal leads to relief of upper airway obstruction and termination of the obstructive event and this arousal has been shown to be accompanied by jaw closure in adults with OSAS [3]. Moreover, an abrupt decrease in pulse transit time (PTT) of at least $10 \mathrm{~ms}$ is recognized as a surrogate for EEGarousal at the end of RE in adults and in children [4, 5].

We present here the temporal association between mandibular movements (MMs) and the changes in PTT in response to OSAS events followed by EEG-arousals during PSG in a pre-school child with upper airway obstruction who was referred for preoperative evaluation. The effects of adenotonsillectomy on MMs and PTT are shown.

\section{CASE REPORT}

A 3-year old boy was evaluated in the otorhinolaryngology clinic for repetitive rhinitis episodes and loud

*Address correspondence to this author at the Sleep Laboratory Pneumology Department - Clinique et Maternité Sainte-Elisabeth, Place Louise Godin, 15 - 5000 Namur, Belgium; Tel: +32 (0) 4955026 08;

Fax: +32 (0) 815707 84; E-mail: martinot.j@ scarlet.be snoring. His mother described apneas during sleep and mouth breathing and dysphagia to solids. Body mass index (BMI) was normal at $15.2 \mathrm{~kg} / \mathrm{m}^{2}$. Upper airway evaluation revealed enlarged pharyngeal tonsils and marked bilateral adenoidal hypertrophy (grade III). He underwent PSG and was diagnosed with severe OSAS (28.8 ApneaHypopnea/hour) (Fig. 1A).

The signals were acquired with a polysomnograph (Dream ${ }^{\circledR}$ Medatec-Brussels-Belgium). A midsagittal sensor (Brizzy ${ }^{\circledR}$ Nomics-Liege-Belgium) measured the distance in $\mathrm{mm}$ between two resonant circuits placed on the forehead and on the chin. The relative position of the mandible was recorded with a resolution of $0.1 \mathrm{~mm}$. The sensors are connected to the electronic module where the signal is digitalized at a sampling frequency of $10 \mathrm{~Hz}$ before transmission into the polysomnograph.

The position of the mandible within the breathing frequency band $(0.25-0.6 \mathrm{~Hz})$ was recorded and peak-to-peak amplitude of the mandibular displacement was measured as previously described [3]. The signal was processed as following: the more negative the signal, the lower the mandible and the greater was mouth opening. PTT was measured on a beat by beat basis as the interval between the $\mathrm{R}$-wave to a threshold on the pulse waveform representing $50 \%$ of its height [6].

Fig. (1B) shows that the mandibular position is lowering (the mouth is opening) during the first 2 hours of sleep; this is depicted by the trend of its mean position (calculated over 10 seconds) which is synchronous with increasing PTT trend.

Four variable MMs patterns were sequentially identify ied during the first two hours of the recording; Pattern 1-high 


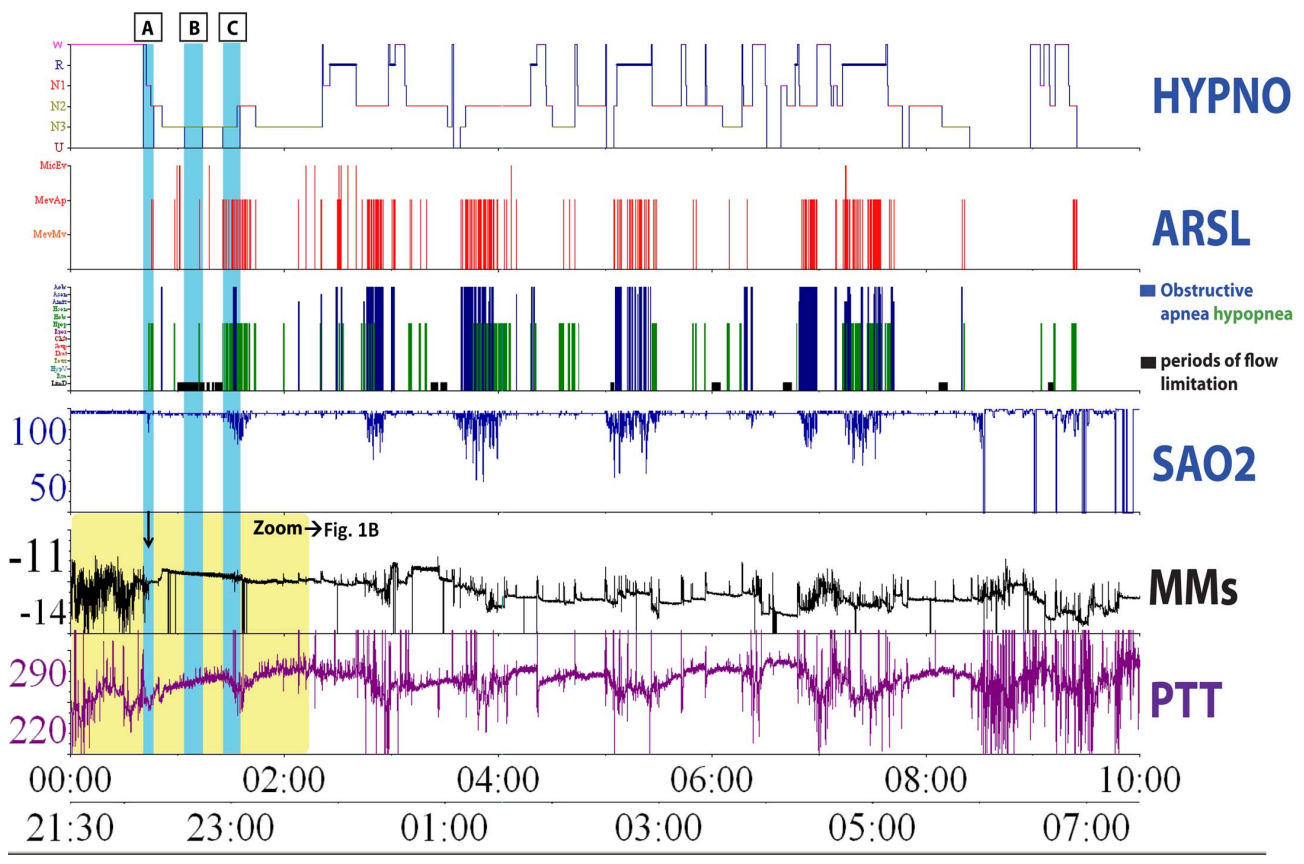

A

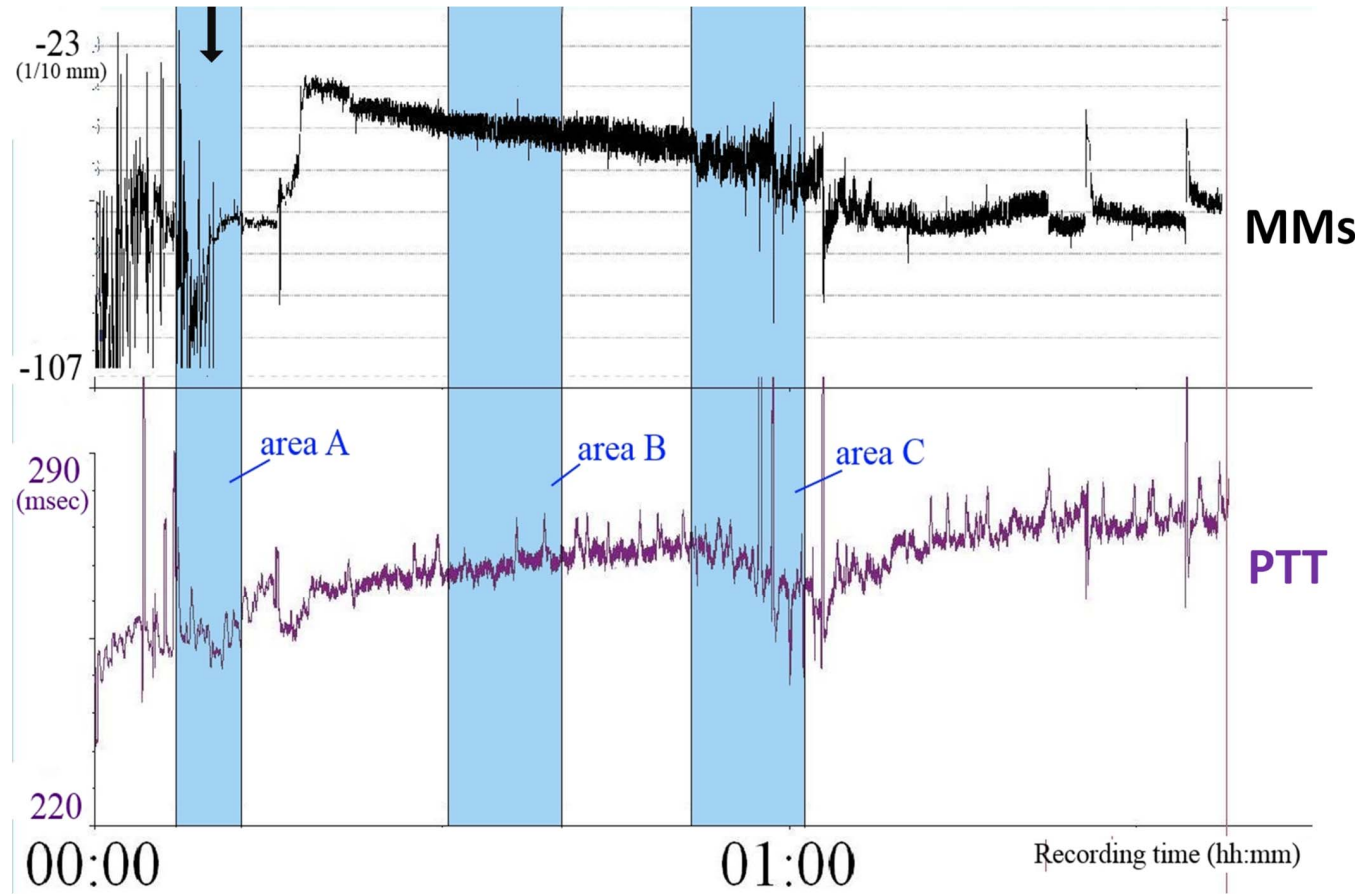

B

Fig. (1). Overnight PSG (Fig. 1A) of a child showing an AHI of 28.8/hour with from the top of the fragment, the hypnogram (sleep stages), the EEG-arousals, the obstructive hypopneas and apneas, each event being represented as a vertical red, green and blue vertical line respectively, and the oximetry, the mandibular position (MMs) and, the pulse transit time (PTT). Periods of flow limitation are shown as horizontal black bars. The highlighted area focuses on the first two hours recording. During this period of time, the trends of the mandibular movements and the PTT were calculated as the average over $10 \mathrm{sec}$. The results are showed before (Fig. 1B) and after adenotonsillectomy (Fig. 3A and $\mathbf{B})$.

Three blue frames (areas A, B and C) in Fig. (1B) are depicted to exhibit the patterns of the mandible behavior enlarged in Fig. (2).

In the area A, the black arrow marks the sleep start. The same is shown in Fig. (1A). The mandible is lowering about $2 \mathrm{~mm}$ during a period of flow limitation as the PTT is increasing (between the two red arrows). The thickness of its signal trace is due to the increase in the peak to peak amplitude movement (area B).

The $r$ Pearson coefficient of a point to point regression (after resampling the PTT at the same frequence used to sample the mandibular position) between the signals of the mandible and PTT is $0.65(\mathrm{P}<0.001)$ during the period of area $\mathrm{C}$. 

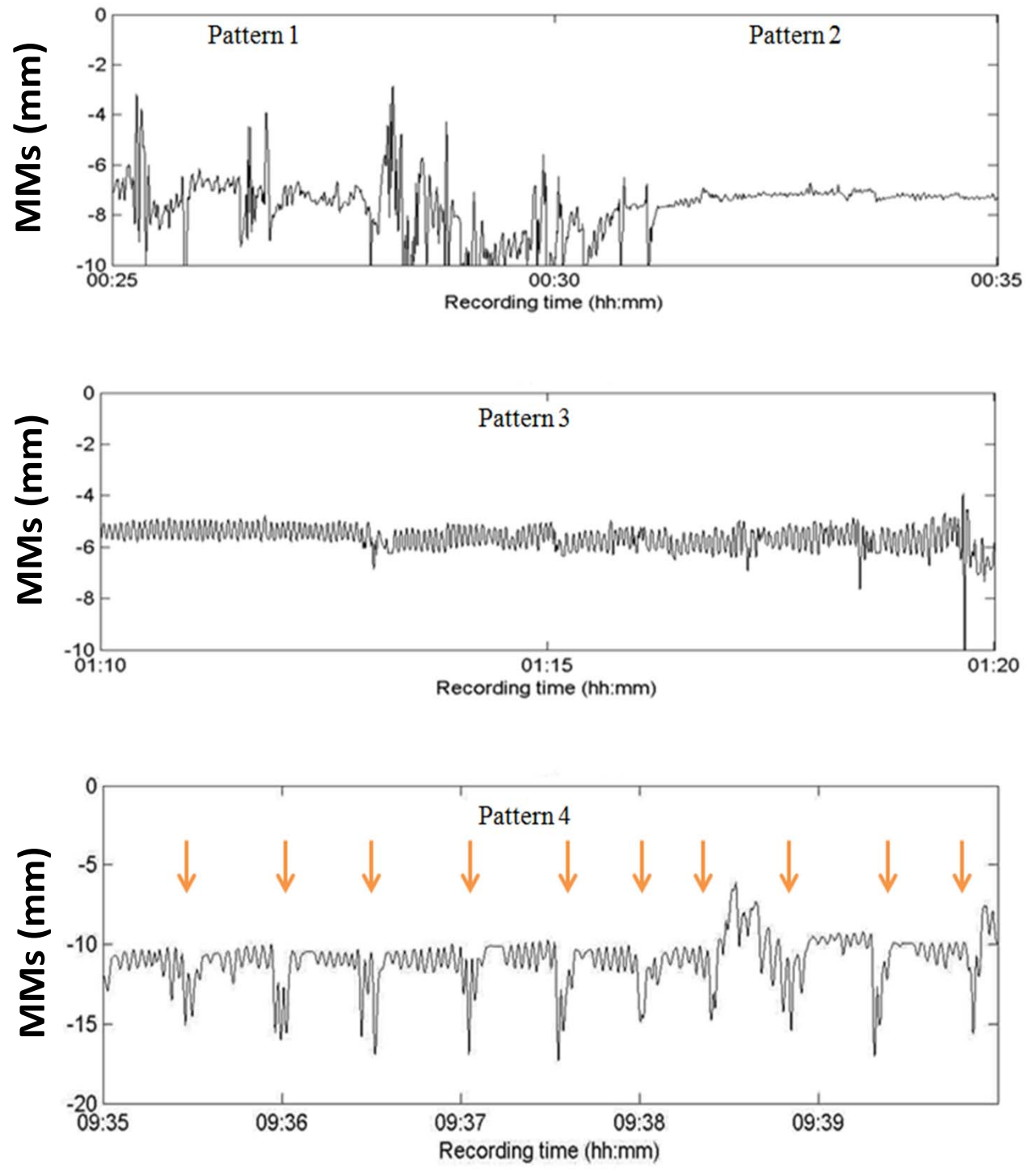

Fig. (2). Mandibular movements as raw data acquired with the magnetic distance-meter corresponding to the blue areas depicted in Fig. (1B): area "A" when awake (pattern 1) and then asleep (pattern 2), area "B" when flow limitation is observed on the nasal flow trace (pattern 3 ) and area " $\mathrm{C}$ " when obstructive apneas or hypopneas are recorded (pattern 4). The arrows indicate concomitant cortical arousals on EEG in relationship with a sharp movement of the mandible.

and unpredictable MMs when still awake corresponding to rapid and discoordinated mandibular activity, Pattern 2stable and minimal mandibular openings in the first part of sleep stage N3 sleep with low amplitude of movements (less than $0.5 \mathrm{~mm}$ ), Pattern 3- MMs of larger amplitude driven by the respiratory effort during a period of flow limitation and lastly Pattern 4- during obstructive hypopneas or apneas, when the position signal of the mandible was interrupted due to EEG-arousals (one or more successive MMs greater in amplitude $>2 \mathrm{~mm}$ to a clenched mouth position shown in Fig. 2).

The patient underwent adenotonsillectomy following which the mother reported significant improvement in snoring, sleep and daytime functioning. A postoperative PSG was performed three months after the surgery to assess resolution of OSAS. The PSG confirmed a marked reduction of the apnea-hypopnea index (AHI) from 28.8 to 4/hour and EEG-arousal index from 27 to 14.8/hour (Fig. 3A). PTT and MMs became much less variable over time (Fig. 3B).

\section{DISCUSSION}

The main finding is that the mid-sagittal position of the mandible varied with breathing frequency, with the peak-topeak excursion changing with respiratory effort intensity and accompanied by increases of PTT during periods of RE in OSAS [5]. The more the mandible lowered, the more PTT lengthened reflecting effort to expand the upper airway (see 


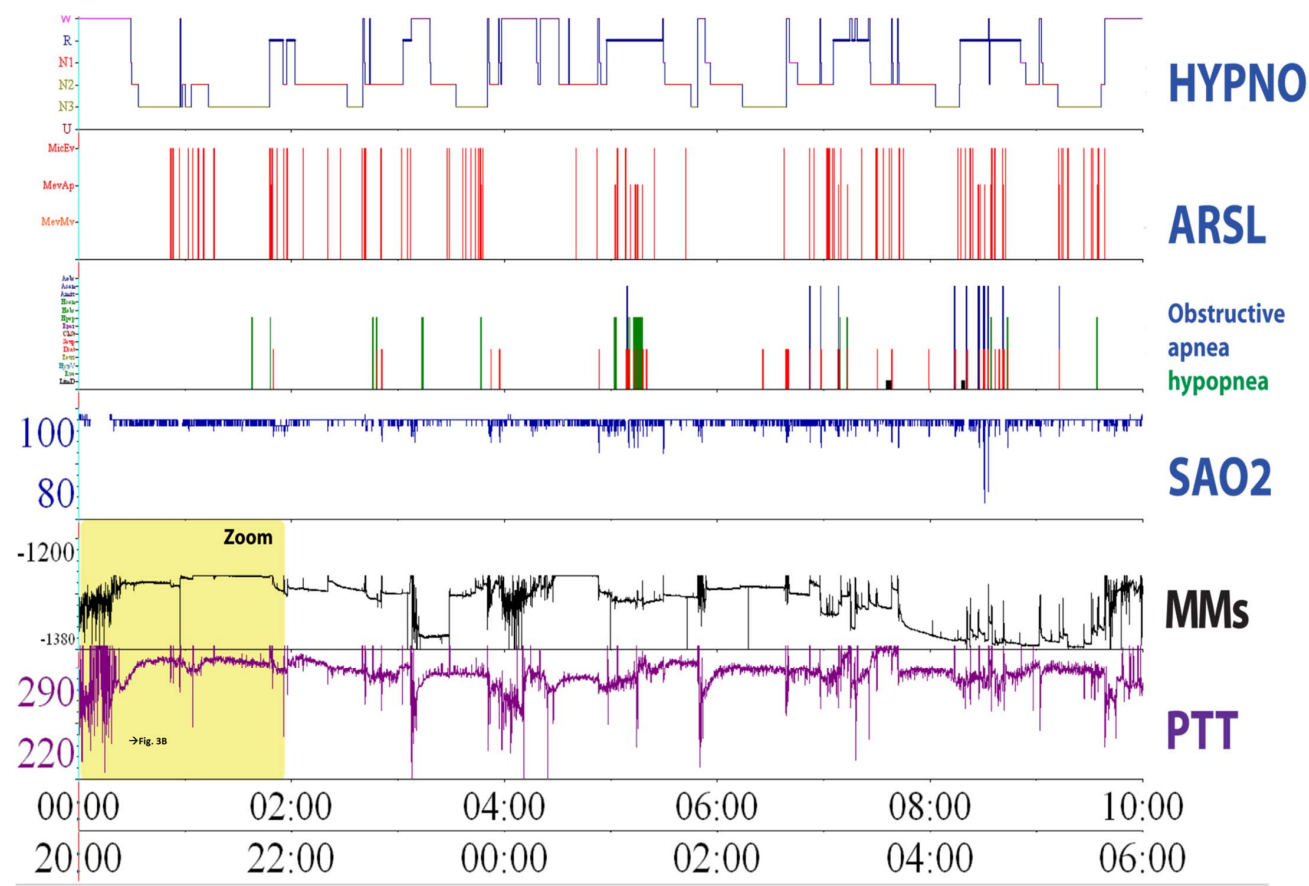

$\mathbf{A}$

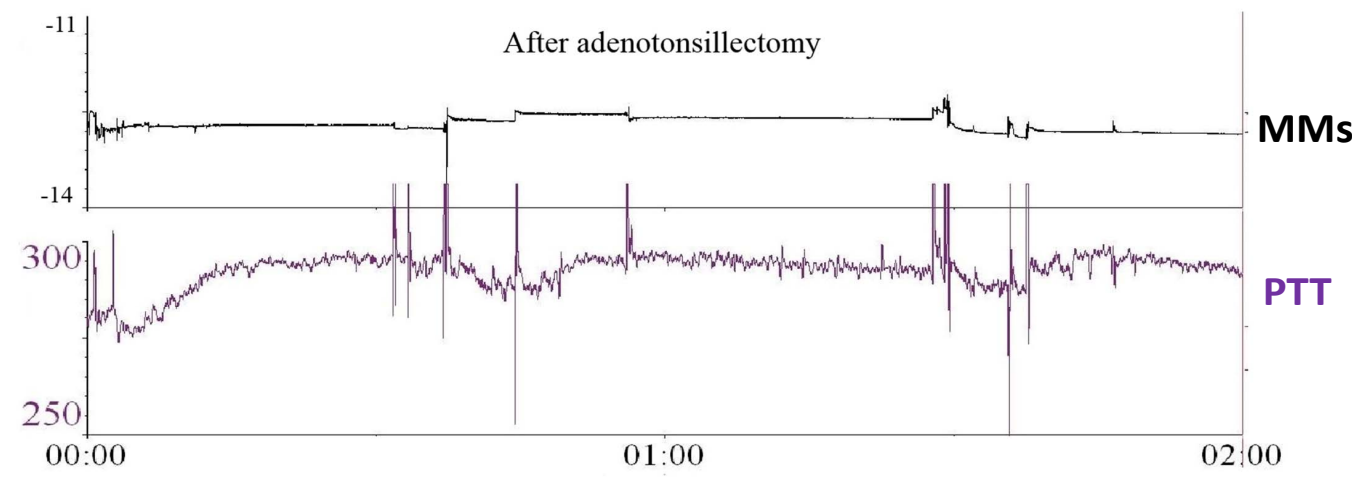

B

Fig. (3). Overnight PSG (Fig. 3A) after adenotonsillectomy and changes observed in the mandibular movements and PTT trends (Fig. 3B).

the respective signal trends in Fig. 1B). Mandibular lowering is produced by geniohyoid and mylohyoid activation [7, 8] opening the mouth thereby allowing mouth breathing [9].

Abrupt decrease in PTT marked the RE related arousals $[5,6,10]$. The occurrence of a cortical arousal terminating the obstructive events was accompanied by the normalization of PTT along with a sudden and sharp closing movement of the mandible (see Fig. 1B area C). This quick closure of the jaw was previously described in adult OSA as an arousal signal $[3,11]$.

$\mathrm{RE}$ detection is critical during sleep to prevent from misclassification of obstructive event as central and to track flow limitation. Beyond oesophageal manometry which is rarely used in clinical practice, only respiratory inductance plethysmography is recommended for detection of RE in adults and in children in the last update of the 2007 AASM manual for the scoring of sleep and associated events [2]. However, this methodology could be insensitive to weak RE and other methods have to be explored to detect RE during sleep [12].

Adenotonsillectomy relieved upper airway obstruction and related RE. This is highlighted with PTT trend remaining unchanged overtime and a marked AHI decrease Correspondingly, mandibular movements disappeared (Fig. 3B). This case report is one example from a series of observations we have made on other patients with similar findings that we are currently analyzing. 
In conclusion, the position of the mandible is a novel additional marker for respiratory effort, and could also help to confirm relief of upper airway obstruction after adenotonsillectomy.

\section{CONFLICT OF INTEREST}

The authors confirm that this article content has no conflicts of interest.

\section{ACKNOWLEDGEMENTS}

The authors wish to thank Dr Philip Silkoff who revised the article critically for intellectual content.

The secretarial assistance of Dr Nam Ledong and Ms Liesbeth Orij were greatly appreciated.

\section{REFERENCES}

[1] Guilleminault C, Pelayo R. Sleep-disordered breathing in children. Ann Med 1998; 30(4): 350-6.

[2] Berry RB, Budhiraja R, Gottlieb DJ, et al. American Academy of Sleep Medicine. Rules for scoring respiratory events in sleep: update of the 2007 AASM Manual for the Scoring of Sleep and Associated Events. Deliberations of the Sleep Apnea Definitions
Task Force of the American Academy of Sleep Medicine. J Clin Sleep Med 2012; 8(5): 597-619.

[3] Senny F, Destiné J, Poirrier R. Mid jaw movements analysis for the scoring of sleep apneas and hypopneas. IEEE Trans Biomed Eng 2008; 55: 87-95.

[4] Argod J, Pépin JL, Lévy P. Differentiating obstructive and central sleep respiratory events through pulse transit time. Am J Respir Crit Care Med 1998; 158: 1778-83.

[5] Katz ES, Lutz J, Black C, Marcus CL. Pulse transit time as a measure of arousal and respiratory effort in children with sleepdisordered breathing. Pediatr Res 2003; 53: 580-8.

[6] Pépin JL, Delavie N, Pin I, et al. Pulse transit time improves detection of sleep respiratory events and microarousals in children. Chest 2005; 127: 722-30.

[7] Hollowell DE, Suratt PM. Mandible position and activation of submental and masseter muscles during sleep. J Appl Physiol 1991; 71: 2267-73.

[8] Wiegand DA, Latz B, Zwillich W, Wiegand L. Geniohyoid muscle activity in normal men during wakefulness and sleep? J Appl Physiol 1990; 69: 1262-9.

[9] Tsuda H, Lowe AA, Chen H, Fleetham JA, Ayas NT, Almeida FR. The relationship between mouth opening and sleep stage-related sleep disordered breathing. J Clin Sleep Med 2011; 7(2): 181-6.

[10] Pagani J, Villa MP, Calcagnini G, et al. Pulse transit time as a measure of inspiratory effort in children. Chest 2003; 124: 1487-93.

[11] Kato T, Katase T, Yamashita S, et al. Responsiveness of jaw motor activation to arousals during sleep in patients with obstructive sleep apnea syndrome. J Clin Sleep Med 2013; 9(8): 759-65.

[12] Stats BA, Bonekat W, Harris CD, Offod KP. Chest wall motion in sleep apnea. Am Rev Respir Dis 1984; 130: 59-63.

Received: January 07, 2014

Revised: April 05, 2014

Accepted: April 14,2014

(C) Martinot et al.; Licensee Bentham Open.

This is an open access article licensed under the terms of the Creative Commons Attribution Non-Commercial License (http://creativecommons.org/licenses/by$\mathrm{nc} / 3.0 /)$, which permits unrestricted, non-commercial use, distribution and reproduction in any medium, provided the work is properly cited. 